\title{
BMJ Open What older adults and their caregivers need for making better health-related decisions at home: a participatory mixed methods protocol
}

\author{
Claudia Lai, ${ }^{1,2}$ Paul Holyoke, ${ }^{2}$ Karine V Plourde, ${ }^{1,3}$ Simon Décary, ${ }^{1,3}$ \\ France Légaré (D) ${ }^{1,3}$
}

To cite: Lai C, Holyoke $P$, Plourde KV, et al. What older adults and their caregivers need for making better health-related decisions at home: a participatory mixed methods protocol. BMJ Open 2020;10:e039102. doi:10.1136/ bmjopen-2020-039102

- Prepublication history and additional materials for this paper is available online. To view these files, please visit the journal online (http://dx.doi. org/10.1136/bmjopen-2020039102).

Received 04 April 2020 Revised 19 0ctober 2020 Accepted 20 0ctober 2020

Check for updates

(c) Author(s) (or their employer(s)) 2020. Re-use permitted under CC BY-NC. No commercial re-use. See rights and permissions. Published by BMJ.

${ }^{1}$ Tier 1 Canada Research in Shared Decision Making and Knowledge Translation, Université Laval Primary Care Research Centre (CERSSPL-UL), Quebec City, Quebec, Canada

${ }^{2}$ SE Research Centre, SE Health, Markham, Ontario, Canada

${ }^{3}$ Department of Family Medicine and Emergency Medicine,

Université Laval, Quebec City, Quebec, Canada

Correspondence to

Dr France Légaré;

france.legare@mfa.ulaval.ca

\section{ABSTRACT}

Introduction Shared decision making is an interpersonal process whereby healthcare providers collaborate with and support patients in decision-making. Older adults receiving home care need support with decision-making. We will explore what older adults receiving home care and their caregivers need for making better health-related decisions. Methods and analysis This two-phase sequential exploratory mixed methods study will be conducted in a pan-Canadian healthcare organisation, SE Health. First, we will create a participant advisory group to advise us throughout the research process. In phase 1 (qualitative), we will recruit a convenience sample of 15-30 older adults and caregivers receiving home care to participate in openended semi-structured interviews. Phase 1 participants will be invited to share what health-related decisions they face at home and what they need for making better decisions. In phase 2 (quantitative), interdisciplinary health and social care providers will be invited to answer a webbased survey to share their views on the decisional needs of older adults and their caregivers. The survey will include questions informed by findings from qualitative interviews in phase 1, and a workbook for assessing decisional needs based on the Ottawa Decision Support Framework. Finally, qualitative and quantitative results will be triangulated (by methods, investigator, theory and source) to develop a comprehensive understanding of decision-making needs from the perspective of older adults, caregivers and health and social care providers. We will use the quality of mixed methods studies in health services research guidelines and the Checklist for Reporting the Results of Internet ESurveys checklist.

Ethics and dissemination Ethics approval was obtained from the research ethics boards at Southlake Regional Health Centre and Université Laval. This study will inform the design of decision support interventions. Further dissemination plans include summary briefs for study participants, tailored reports for home care decision makers and policy makers, and peer-reviewed publications.

Trial registration number NCT04327830.

\section{INTRODUCTION}

With our ageing population, an increasing number of older adults are faced with
Strengths and limitations of this study:

- This study will be the first to use a sequential mixed methods approach to assess decisional needs of older adults receiving home care and their caregivers from the perspective of older adults, caregivers and interdisciplinary health and social care providers.

- Our participatory approach, involving participants as research partners, aims to produce findings that better reflect the decisional needs of those whom the research is meant to benefit.

- Qualitative findings reflecting the decisional needs of a group of older adults and caregivers in Ontario $(n=15-30)$ will be tested on a larger group of interdisciplinary health and social care providers who provide home care services across different provinces $(n=500)$ to strengthen and enhance our multiperspective findings.

- Using a convenience sample of participants may introduce selection bias.

- Completion of the web-based survey in English only may omit unique perspectives from non-English speaking health and social care providers.

important and often difficult health-related decisions as they grow older in their own homes. ${ }^{1-3}$ These decisions may be about medication, surgery, safety, care transitions, housing transitions, advance care planning and medical assistance in dying. ${ }^{4-6}$ However, making health-related decisions may often lead to significant decisional conflict, or the feeling of personal uncertainty over which options are best for a specific individual facing a specific situation. In most real-world scenarios, it is difficult to establish one clear best option, and thus many health-related decisions are preference-sensitive.

Shared decision making (SDM) refers to an interpersonal, interdependent process whereby patients and their healthcare providers relate to and influence each other 
as they collaborate in making health-related decisions together. ${ }^{78} \mathrm{SDM}$ has been associated with improving wellbeing, independence and experiences of the health and social care system. ${ }^{9-11}$ SDM aims to engage patients to play an active role in decisions concerning their health, the ultimate goal of person-centred care. SDM rests on the best evidence of the risks and benefits of all the available options. In the case of older adults, health decisions are more complex and often involve caregivers. In fact, when older adults suffer from cognitive deficits, their caregivers may be asked to make decisions for them. ${ }^{12}$ Older adults may also experience multimorbidities ${ }^{5}$ and polypharmacy, ${ }^{13} 14$ resulting in an unmanageable burden of treatment. Moreover, they may be cared for by a large number of healthcare providers. Thus, techniques that enable older adults and caregivers to prioritise main issues and then adequately weigh the risks and benefits associated with treatment choices are all the more important. ${ }^{5}$ Equally important is establishing a care culture in which the values and preferences of older adults are sought and their opinions valued. Programmes most likely to effectively promote SDM with older people are those that allow them to feel respected, understood and give them the confidence to engage in SDM. ${ }^{15}$

In recent years, there has been an increased interest in a team-based approach to SDM. ${ }^{16}$ Indeed, health and social care providers, such as personal support workers (PSW), physiotherapists (PT), occupational therapists (OT), nurses and other clinicians, can together play an important role in supporting older adults and their caregivers with decision making. An inter-professional approach to SDM has several advantages: teams contribute different knowledge and skills to the decision-making process, thus producing more feasible and sustainable decisions. From the older adult's standpoint, the interprofessional approach fosters engagement in decisionmaking across the continuum of care. Thus, it has the potential to improve the quality of care and decision support provided, as it would be performed in a more integrated manner. Finally, it bridges the gap between professionals from various health disciplines and patients and their families, thereby reducing the silos. ${ }^{17}$ Research to demonstrate how inter-professional teams of health and social care providers can collaboratively support decision-making in older adults have shown that an interprofessional approach to SDM is acceptable and feasible in the home care sector. ${ }^{18} 19$ This is crucial evidence as a recent pan-Canadian survey reported that Canadians experienced relatively low levels of SDM. ${ }^{20}$ In particular, older adults receiving home care are less likely to experience SDM when faced with health and social care decisions than any other sociodemographic group. ${ }^{20}$

A building block for increasing SDM among older adults receiving home care is a decisional needs assessment. ${ }^{21}$ Understanding and assessing the decision making needs not only of patients, but also of their caregivers and health providers in home care, will inform us about ways to better engage older adults and their caregivers in SDM, and how healthcare providers' can better support them to make decisions together that best reflects their preferences. Numerous studies have assessed the decisional needs of specific populations, such as patients needing complex care, ${ }^{22}$ those with heart disease,${ }^{23}$ advanced kidney disease ${ }^{24}$ and psoriasis. ${ }^{25}$ However, no studies that assess the decisional needs of older adults receiving home care were identified in a recent systematic review. ${ }^{26}$ Therefore, we seek to identify what older adults receiving home care and their caregivers need for making better decisions at home.

\section{Study objectives}

The study will explore in phase 1: (1) decisions facing older adults receiving home care and their caregivers; (2) what older adults receiving home care and their caregivers need for making better decisions at home (decisions that are informed by the best evidence and that are congruent with what matters to them); and in phase 2: (3) what interdisciplinary health and social care providers need for better supporting their clients to make better decisions at home.

\section{METHODS AND ANALYSIS \\ Design and setting}

The proposed study will use a sequential exploratory mixed methods design, which involves a first phase of qualitative data collection and analysis followed by a second phase of quantitative data collection and analysis that builds on the previous phase (figure 1) ${ }^{27}$ Thus, quantitative data and results will assist in the interpretation of qualitative findings. In phase 1 , we will collect qualitative data from open-ended semi-structured interviews with older adults receiving home care services and their caregivers in Ontario. In phase 2, we will collect quantitative data from web-based surveys completed by interdisciplinary health and social care providers who provide home care services across different provinces (Quebec, Ontario, Alberta and British Columbia). Qualitative and quantitative findings will subsequently be integrated to enhance our understanding of what is needed for SDM from the perspective of older adults, caregivers and care providers. We applied the following guidelines in the development of this protocol: (1) criteria reported in an assessment of the quality of mixed methods studies in health services research by O'Caithan $e t a l^{28}$ and (2) the Checklist for Reporting the Results of Internet E-Surveys (CHERRIES) ${ }^{29}$ The criteria reported by O'Caithan et al was used for describing the design for our health services research, and how we will integrate qualitative and quantitative data between the two phases. The items on the CHERRIES checklist was used to design the online survey for phase 2 of the study, including considerations for developing and pretesting the survey, survey administration and response rates.

\section{Theoretical framework}

Ottawa Decision Support Framework

The Ottawa Decision Support Framework (ODSF) will inform our preliminary analytical framework, ${ }^{21}$ which will 


\begin{abstract}
Semi-structured open ended interviews with 15 to 30 older adults (and caregivers) Qualitative data analysed using thematic analysis

Initial coding done independently by two coders according to initial thematic map Collate codes into emergent themes

Team meetings for consensus on codes, themes and categories

Modified thematic map grounded in both the literature and data
\end{abstract}

Phase 2 (quantitative data)
Quantitative data collected from survey of 500 health and social care providers
Descriptive statistics of (minimum, maximum, mean, SD)
Group comparisons (e.g., t-test, Mann-Witney, ANOVA)

\title{
Integrate qualitative and quantitative findings
}

List the findings from each component of study and determine "meta-themes" that cut across findings from the different methods, as well as where a theme arises from one data set and not another

Figure 1 Study design. ANOVA, analysis of variance.

be modified and improved on with emergent concepts and themes. The ODSF is a well-established framework for assessing what individuals need for making informed and value-congruent decisions where multiple options must be considered, that is, decisions informed by the best evidence available, and by what matters most to them. A better understanding of the decisional needs of older adults receiving home care and their caregivers is important for designing SDM interventions to support those who feel uncertain about options (decisional conflict), and/or who lack knowledge, or have expectation deficits, unclear values or insufficient supports and resources. ${ }^{21}$ The ODSF has been used in previous studies including older adults, ${ }^{26}$ and will enhance our understanding in the understudied area of decisional needs among older adults in home care.

\section{Transformative perspective}

Adopting a transformative perspective, we will critically examine the decisional needs of older adults and their caregivers with an awareness of the complex relationship between knowledge and power. ${ }^{30} 31$ Decision-making in the home care context can be affected by power imbalances between healthcare providers and patients, cultural factors, disabilities and complex care needs. ${ }^{32-35}$ Understanding how the decisional needs of older adults and caregivers are affected by systemic and institutional barriers can inform interventions to strengthen their ability to participate in SDM (eg, that ensure their voices are heard). The transformative perspective has been used for needs assessments in marginalised communities, ${ }^{36}$ and is consistent with the Canadian Institute of Health Research (CIHR) efforts 'to help transform the role of patient from a passive receptor of services to a proactive partner who helps shape health research and, as a result, healthcare'? ${ }^{37}$

\section{Patient and public involvement}

One older adult, one caregiver and two care providers will participate as research partners who will be involved throughout the research process. The research partners will form a participant advisory group to guide the study design and conduct (including reviewing the study protocol, the interview guide and survey questions) and help with interpretation of findings. Training and tools will be provided as required so that they can actively collaborate as part of the research team. ${ }^{38}$ This research partnership is consistent with the CIHR patient-oriented research approach that aims to 'ensure that studies focus on patient-identified priorities, which ultimately leads to better patient outcomes'.

\section{Participants and recruitment}

Older adults and their caregivers

Phase 1 participants will include: (1) older adults who are receiving or have received home care services and (2) caregivers of older adults who are receiving or have received home care services. As ageing is not a uniform process across populations, participants in the older adult group will consist of individuals who self-identify as older adults with home care experience. This approach aims to include individuals who share a worldview as an older adult. Participants under 18 years of age, such as caregivers caring for their grandparents, are excluded from this study as they may have additional concerns that can be further explored in future studies.

Our recruitment method includes: (1) sending recruitment notices through professional and patient networks 
at SE Health, one of Canada's largest social enterprises providing home care services (SE Health's wiser advisors platform, SE research team's Twitter and Facebook websites, SE Health service provider contacts); and (2) posting recruitment notices in public places (such as grocery stores, community centres, libraries). Participants will also be recruited using a snowball strategy whereby participants may suggest other participants for recruitment.

We are expecting to interview about 15-30 participants to reach saturation where sufficient depth of understanding has been achieved in relation to emergent theoretical categories. ${ }^{39-41}$

We will purposefully select participants with different backgrounds (in terms of age, gender, native/non-native English speaking, racial and/or ethnic backgrounds, disability status and medical conditions) for maximum variability. ${ }^{42}$ This selection strategy aims to identify decisional needs that might be common among participants with different backgrounds, as well as unique or diverse variations that might relate to certain conditions or contexts (eg, decisional needs unique to older adults under 65 years of age who may not be eligible for public drug coverage due to their age). Thus, we aim to identify both the decisions older adults find most difficult, and the kinds of decisional support they might need depending on their decision-making contexts, cultures and preferences. Potential participants will be informed that their participation is voluntary, and that their responses will be kept confidential. Informed consent will be sought from all participants. Participants will be offered a $\$ 20$ gift card as a token of appreciation for their time and input, if they wish to receive it. To the best of our knowledge, SE Health does not currently have specific rules or decision-support guidelines for clients and caregivers that might influence results. We cannot speak to other home care companies that may have provided care to participants in this study.

\section{Interdisciplinary health and social care providers}

Phase 2 participants will include front-line interdisciplinary health and social care providers who provide in-person home care services to older adults. These include PSW, healthcare aides or préposés aux bénéficiaires; registered nurse assistants; registered practical nurses or licensed practical nurses; registered nurses; advance practice nurses (e.g., nurse practitioners, clinical nurse specialists); OT; PT and other providers. Their client loads, hours per visit and number of visits per client can vary. As response rates in online surveys differ from traditional survey methods, ${ }^{29}$ we are targeting a sample size of up to $10 \%$ of the 5000 health and social care providers who work at SE Health (eg, up to 500 participants depending on local operational priorities). The actual number of participants will depend on operational priorities at the launch of the survey. Potential participants will be informed that their participation is voluntary, and that their responses will be kept confidential. Their decision to participate in the study or not will have no impact on their work in the organisation. Participants will be offered a choice to enter a draw for a $\$ 20$ gift card (100 gift cards in total) as a token of appreciation for their time and input.

\section{Data collection}

Data collection in phase 1 and phase 2 of the study will be guided by the following research questions:

- What health-related decisions are older adults receiving home care and their caregivers faced with at home?

- What do older adults receiving home care and their caregivers need for making better decisions at home?

- What do interdisciplinary health and social care providers who provide home care services to older adults need for better supporting their clients with making decisions?

In phase 1, qualitative data will be collected from open-ended semi-structured interviews conducted with older adults who have received or are receiving home care services, and their caregivers. Adopting standardised questions based on the ODSF, ${ }^{21}$ the interview guide (see online supplemental file 1) includes questions on what important and difficult decisions they face at home, what makes these decisions difficult, how they feel when making these decisions, and what they need for making better decisions. The questions will be used as probes to encourage discussion. According to the ODSF, decisional needs may be related to decisional conflict, a feeling of lacking knowledge, expectations, values clarity, and a lack of support and/or resources, which can be specific to the type of decision as well as to the characteristics of older adults and their caregivers. ${ }^{21}$ As such, our qualitative approach aims to gain an in-depth understanding of decision making needs in the home care setting.

The interviews are expected to take about $60 \mathrm{~min}$, and will be exploratory and open ended to encourage conversation about their decision making process, which can include difficulties they face in their decision making process, how they negotiated decisions and with whom, what supports (or pressures) did they receive, and how complex and changing health needs might affect their process. All interviews will be recorded and transcribed verbatim. Interviews will be conducted at the participant's home, or an alternative location of his/her choosing.

In phase 2, quantitative data will be collected from webbased surveys answered by health and social care providers who work at SE Health across provinces (Quebec, Ontario, Alberta and British Columbia). The survey (see online supplemental file 2) will include: (1) Section Aabout you; (2) Section B-your views on decisions facing older adults (and their caregivers) in home care settings; and (3) Section C-your views on the decision-making needs of older adults (and their caregivers) in home care settings. The web-based survey is expected to take about 5-10 min and can be completed on mobile devices or a computer. The survey will be pretested for readability 
and pilot tested in a small group of participants before launching more widely across the SE Health organisation.

\section{Analysis}

Phase 1 qualitative data will be systematically analysed using thematic analysis, which is a method for identifying, analysing and reporting patterns (themes) within data. ${ }^{43}$ N-Vivo V.12 Plus software will be used to support the analysis. Thematic analysis allows for the systematic coding of qualitative data according to a preliminary thematic map, which will be informed by rich concepts from the ODSF. ${ }^{21}$ This approach is flexible to allow the thematic map to be expanded on with emergent concepts and themes using a transformative perspective. Our thematic analysis will follow five steps. ${ }^{43}$ First, we will familiarise ourselves with the data by reading and re-reading the transcripts and noting down initial ideas. Second, the transcripts will be independently coded by two coders across the entire data set in a systematic fashion, collating data relevant to each code. Third, we will collate codes into emergent themes, gathering all data relevant to each theme. We will discuss the codes and themes as a team and come to a consensus. Discrepancies will be discussed until a consensus is reached. Fourth, we will review the themes in relation to the coded extracts and the entire data set to generate a modified thematic map of the analysis that is grounded in both the literature and data. Finally, we will refine the specifics of each theme and generate a report on the thematic map with clear definitions of each theme. We will select vivid and compelling extracts, referring back to the analysis and literature to produce a report of the analysis.

Phase 2 quantitative data collected from the web-based survey will be analysed using SPSS Statistics 27. Descriptive statistics will be used to analyse the survey data (eg, frequencies, weighted average, median, variance), as well as other statistical tools (eg, Mann-Witney test to analyse the ordinal data (5-point Likert scale) and t-tests for group comparisons). Qualitative and quantitative findings will subsequently be integrated to report on the decisional needs of older adults receiving home care and their caregivers from multiple perspectives. Our final report will be shared with phase 1 participants who expressed an interest in receiving study findings for member checking to strengthen results.

We will use multiple types of triangulation to enhance the reliability of our findings: (1) method triangulation: data collected from semi-structured interviews will be triangulated with data collected from online surveys; (2) investigator triangulation: data will be individually coded by two coders and discussed with our research team to mitigate the risks of bias that might come from the analysis of individual researchers and add breadth to the study; (3) theory triangulation: data will be initially coded using a thematic map informed by well-established concepts from the ODSF, which will be expanded on with emergent themes from a transformative perspective; and (4) data source triangulation: data collected from interdisciplinary health and social care providers will be used to triangulate data from older adults and their caregivers, and vice versa, to test and improve reliability of our findings. ${ }^{42} 44$

This study will be among the first to assess the decisional needs of older adults receiving home care and their caregivers. This participatory study will use a sequential mixed methods design to assess the decisional needs of older adults and their caregivers from their own perspectives and that of numerous different types of home care providers.

\section{ETHICS AND DISSEMINATION \\ Ethics}

Ethics approval was obtained from the research ethics boards at Southlake Regional Health Centre in Ontario and Université Laval in Québec. Participants will be provided with an information sheet on the study and will have time to ask questions about the study before enrolment. Informed consent will be sought from all study participants prior to their participation.

\section{Dissemination}

We will disseminate results of the semi-structured interviews and survey in a peer-reviewed publication and at conferences. Our dissemination plan includes: summary briefs for study participants; summary briefs for posting on our social media platforms; tailored reports for home care decision makers and policy makers to improve the resources provided to support older adults with their decision making at home; reports for our Tier 1 Canada Research Chair in Shared Decision Making and Knowledge Translation team to guide the development of robust decision support interventions for transforming how health and social care providers work with older adults and their caregivers to make decisions together.

\section{Twitter France Légaré @SDM_ULAVAL}

Acknowledgements We would like to acknowledge the study advisors, who provided feedback on the protocol design and recruitment process, as well as the wording of the survey instrument, and Dr Louisa Blair, who provided invaluable editorial feedback to improve the writing of this manuscript.

Contributors The protocol was developed by CL under the mentorship of FL (Tier 1 Canada Research Chair in Shared Decision Making and Knowledge Translation) and PH (applied researcher and expert in the area of home care). KVP and SD were major contributors in the writing of the manuscript. All authors have read and approved the final manuscript.

Funding This work was supported by a CIHR Foundation grant (FDN-159931) aimed at scaling and spreading shared decision for older adults (and caregivers) in the home care setting across Canada, as well as an unrestricted grant from SE Health. SE Health will not be involved in the design of the study, data collection or analysis, interpretation of data, or the writing of any manuscript. FL holds a Tier 1 Canada Research Chair in Shared Decision Making and Knowledge Translation.

Competing interests This study will be conducted in SE Health, one of the largest social enterprises offering home care services across Canada.

Patient consent for publication Not required.

Provenance and peer review Not commissioned; externally peer reviewed.

Supplemental material This content has been supplied by the author(s). It has not been vetted by BMJ Publishing Group Limited (BMJ) and may not have been 
peer-reviewed. Any opinions or recommendations discussed are solely those of the author(s) and are not endorsed by BMJ. BMJ disclaims all liability and responsibility arising from any reliance placed on the content. Where the content includes any translated material, BMJ does not warrant the accuracy and reliability of the translations (including but not limited to local regulations, clinical guidelines, terminology, drug names and drug dosages), and is not responsible for any error and/or omissions arising from translation and adaptation or otherwise.

Open access This is an open access article distributed in accordance with the Creative Commons Attribution Non Commercial (CC BY-NC 4.0) license, which permits others to distribute, remix, adapt, build upon this work non-commercially, and license their derivative works on different terms, provided the original work is properly cited, appropriate credit is given, any changes made indicated, and the use is non-commercial. See: http://creativecommons.org/licenses/by-nc/4.0/.

\section{ORCID iD}

France Légaré http://orcid.org/0000-0002-2296-6696

\section{REFERENCES}

1 Statistics Canada. Home health care and related services [online]. Ottawa: Statitics Canada, 2017. https://www150.statcan.gc.ca/n1/ daily-quotidien/190731/dq190731c-eng.htm

2 Canadian Institute for Health Information. Home care expenditures in the NHEX Database [Technical note on the online]. Ottawa: Canadian Institute for Health Information, 2017. https://www.cihi.ca/sites/ default/files/document/technical_notes_on_home_care-nhex2017en.pdf

3 Statistics Canada. Home health care and related services [online]. Ottawa: Statistics Canada, 2017. https://www150.statcan.gc.ca/n1/ daily-quotidien/190731/dq190731c-eng.htm

4 Garvelink MM, Jones CA, Archambault PM, et al. Deciding how to stay independent at home in later years: development and acceptability testing of an informative web-based module. JMIR Hum Factors 2017;4:e32.

5 Hoffmann T, Jansen J, Glasziou P. The importance and challenges of shared decision making in older people with multimorbidity. PLoS Med 2018;15:e1002530.

6 Garvelink MM, Groen-van de Ven L, Smits C, et al. Shared decision making about housing transitions for persons with dementia: a fourcase care network perspective. Gerontologist 2019;59:822-34.

7 Légaré F, Moumjid-Ferdjaoui N, Drolet R, et al. Core competencies for shared decision making training programs: insights from an international, interdisciplinary working group. J Contin Educ Health Prof 2013;33:267-73.

8 Légaré F, Witteman HO. Shared decision making: examining key elements and barriers to adoption into routine clinical practice. Health Aff 2013;32:276-84.

9 Hibbard JH, Greene J. What the evidence shows about patient activation: better health outcomes and care experiences; fewer data on costs. Health Aff 2013;32:207-14.

10 Shay LA, Lafata JE. Where is the evidence? A systematic review of shared decision making and patient outcomes. Med Decis Making 2015;35:114-31.

11 Veroff D, Marr A, Wennberg DE. Enhanced support for shared decision making reduced costs of care for patients with preferencesensitive conditions. Health Aff 2013;32:285-93.

12 Garvelink MM, Ngangue PAG, Adekpedjou R, et al. A synthesis of knowledge about caregiver decision making finds gaps in support for those who care for aging Loved ones. Health Aff 2016;35:619-26.

$13 \mathrm{Kim}$ J, Parish AL. Polypharmacy and medication management in older adults. Nurs Clin North Am 2017;52:457-68.

14 Maher RL, Hanlon J, Hajjar ER. Clinical consequences of polypharmacy in elderly. Expert Opin Drug Saf 2014;13:57-65.

15 Bunn F, Goodman C, Russell B, et al. Supporting shared decision making for older people with multiple health and social care needs: a realist synthesis. BMC Geriatr 2018;18:165.

16 Dogba MJ, Menear M, Stacey D, et al. The evolution of an interprofessional shared decision-making research program: reflective case study of an emerging paradigm. Int $\mathrm{J}$ Integr Care 2016;16:4.

17 Légaré $\mathrm{F}$, Stacey $\mathrm{D}$, Brière $\mathrm{N}$, et al. A conceptual framework for interprofessional shared decision making in home care: protocol for a feasibility study. BMC Health Serv Res 2011;11:23.

18 Légaré $\mathrm{F}$, Brière $\mathrm{N}$, Stacey $\mathrm{D}$, et al. Implementing shared decisionmaking in interprofessional home care teams (the IPSDM-SW study): protocol for a stepped wedge cluster randomised trial. BMJ Open 2016;6:e014023.

19 Adekpedjou R, Haesebaert J, Stacey D, et al. Variations in factors associated with healthcare providers' intention to engage in interprofessional shared decision making in home care: results of two cross-sectional surveys. BMC Health Serv Res 2020;20:203.

20 Haesebaert J, Adekpedjou R, Croteau J, et al. Shared decisionmaking experienced by Canadians facing health care decisions: a web-based survey. CMAJ Open 2019;7:E210-6.

21 Jacobsen MJ, O'Connor A, Stacey D. Decisional needs assessment in populations: a workbook for assessing patients' and practitioners' decision making needs [online]. Ottawa: University of Ottawa, 2013. https://decisionaid.ohri.ca/docs/implement/population_needs.pdf

22 Poitras M-E, Hudon C, Godbout I, et al. Decisional needs assessment of patients with complex care needs in primary care. $J$ Eval Clin Pract 2020;26:1-14.

23 Blumenthal-Barby JS, Kostick KM, Delgado ED, et al. Assessment of patients' and caregivers' informational and decisional needs for left ventricular assist device placement: implications for informed consent and shared decision-making. J Heart Lung Transplant 2015;34:S336.

24 Loiselle M-C, Michaud C, O'Connor A. Decisional needs assessment to help patients with advanced chronic kidney disease make better dialysis choices. Nephrol Nurs J 2016;43:463.

25 Tan J, Stacey D, Fung K, et al. Treatment decision needs of psoriasis patients: cross-sectional survey. J Cutan Med Surg 2010;14:233-9.

26 Hoefel L, O'Connor AM, Lewis KB, et al. 20th anniversary update of the Ottawa decision support framework Part 1: a systematic review of the decisional needs of people making health or social decisions. Med Decis Making 2020;40:555-81.

27 Creswell JW. Mixed methods procedures. In: Research design. 3rd edn. Thousand Oaks, CA: Sage, 2008: 203-24.

28 O'Cathain A, Murphy E, Nicholl J. The quality of mixed methods studies in health services research. J Health Serv Res Policy 2008;13:92-8.

29 Eysenbach G. Improving the quality of web surveys: the checklist for reporting results of Internet E-Surveys (cherries). J Med Internet Res 2004;6:e34.

30 Mertens DM. Transformative mixed methods research. Qualitat Inquiry 2010;16:469-74.

31 Mertens DM. Transformative mixed methods. Am Behav Sci 2012;56:802-13.

32 Emanuel EJ, Emanuel LL. Four models of the physician-patient relationship. JAMA 1992;267:2221-6.

33 Veinot T. Power to the patient? A critical examination of patient empowerment discourses. In: Harris R, Wathen N, Wyatt S, eds. Configuring health consumers: Health work and imperative of personal responsibility. Hampshire: Palgrave Macmillan, 2010.

34 Chang Y-P, Schneider JK, Sessanna L. Decisional conflict among Chinese family caregivers regarding nursing home placement of older adults with dementia. J Aging Stud 2011;25:436-44.

35 Jull J, Giles A, Boyer Y, et al. The aboriginal women's support center, boyer $\mathrm{Y}$, and stacey D. Cultural adaptation of a shared decision making tool with Aboriginal women: a qualitative study. BMC Med Inform Decision Making 2015;15:1.

36 Jackson KM, Pukys S, Castro A, et al. Using the transformative paradigm to conduct a mixed methods needs assessment of a marginalized community: methodological lessons and implications. Eval Program Plann 2018;66:111-9.

37 CIHR. Strategy for patient oriented research [online]. Available: https://cihr-irsc.gc.ca/e/41204.html [Accessed 10 Feb 2020].

38 Jennings $\mathrm{H}$, Slade $\mathrm{M}$, Bates $\mathrm{P}$, et al. Best practice framework for patient and public involvement (PPi) in collaborative data analysis of qualitative mental health research: methodology development and refinement. BMC Psychiatry 2018;18:213.

39 Miles MB, Huberman AM. Qualitative data analysis: an expanded sourcebook. 2nd SAGE. Thousand Oaks, CA 1994.

40 Guest G, Bunce A, Johnson L. How many interviews are enough? Field methods 2006;18:59-82.

41 Saunders B, Sim J, Kingstone T, et al. Saturation in qualitative research: exploring its conceptualization and operationalization. Qual Quant 2018;52:1893-907.

42 Patton MQ. Qualitative research \& evaluation methods. 3rd edn. Thousand Oaks, CA: Sage Publications, 2002.

43 Braun V, Clarke V. Using thematic analysis in psychology. Qual Res Psychol 2006;3:77-101.

44 Carter N, Bryant-Lukosius D, DiCenso A, et al. The use of triangulation in qualitative research. Oncol Nurs Forum 2014;41:545-7. 